\title{
Echocardiography of the tricuspid valve
}

\author{
Jamahal Luxford ${ }^{1}$, Levi Bassin ${ }^{2}$, Michael D'Ambra ${ }^{3}$ \\ ${ }^{1}$ Department of Anaesthesia and Acute Pain Medicine, St Vincent's Hospital, Melbourne, Australia; ${ }^{2}$ Department of Cardiothoracic Surgery, Royal \\ North Shore Hospital, Sydney, Australia; ${ }^{3}$ Department of Anesthesiology, University of Maryland, Baltimore, MD, USA \\ Correspondence to: Dr. Jamahal Luxford, MBBS, FANZCA. Staff Anaesthetist, Department of Anaesthesia and Acute Pain Medicine, St Vincent's \\ Hospital, Melbourne, Australia. Email: jamahal@hotmail.com.
}

\begin{abstract}
The tricuspid valve (TV), although occasionally considered "neglected" is the subject of renewed and increasing interest. Factors include an awareness that tricuspid value dysfunction is influential in patient outcomes, an improving understanding of valve anatomy and function and evolving techniques available to address tricuspid regurgitation. Tricuspid regurgitation (TR) can be classified as being due to primary diseases of the valve or functional in nature, with the majority being functional. Whilst it was previously believed that such functional TR, resulting from left sided disease, would resolve after correction of the underlying pathology this is now known not to be true. In fact, annular dilatation, TR and right ventricular dysfunction may all continue to progress after successful surgery on the aortic or mitral valve. Although there are many modalities with which to image the TV, this lecture will focus on echocardiography, primarily transesophageal echocardiography (TEE). In every patient undergoing cardiac surgery with TEE, a thorough and systematic examination of the TV structure and function should be performed, utilizing quantitative and qualitative measures with both $2 \mathrm{D}$ and $3 \mathrm{D}$ echocardiography. As the appearance of TR can be significantly influenced by hemodynamic factors, it is essential that echocardiography to investigate TR also be performed in the resting conscious state. Ideally, deciding whether the TV warrants operative attention at the time of planned cardiac surgery should be determined preoperatively based on a high quality transthoracic echocardiography (TTE) and relevant patient and surgical factors. This lecture aims to give an overview of the echocardiographic assessment of the TV, parameters available to grade the severity of TR, and how these may be utilized to assist the surgeon considering intervention. Whilst the surgical management at the extremes of TR (mild or severe) is relatively clear, the ideal intervention in intermediate grades, especially during concurrent left sided surgery remains uncertain and is the subject of ongoing research.
\end{abstract}

Keywords: Echocardiography; tricuspid valve; tricuspid regurgitation; transthoracic echocardiography (TTE)

Submitted Feb 23, 2017. Accepted for publication May 10, 2017.

doi: 10.21037 /acs.2017.05.15

View this article at: http://dx.doi.org/10.21037/acs.2017.05.15

\section{Introduction}

The tricuspid valve (TV) is sometimes considered neglected, even occasionally being referred to as the "forgotten" valve (1). Much of the focus of modern cardiac valvular surgery has been on diseases of the aortic and mitral valves. Even the recent developments in percutaneous technologies have focused on these pathologies. Recently however interest in the TV has been increasing, in part due to evidence that dysfunction of this valve may be more influential in patient outcomes than previously thought. Other factors include: an improved understanding of the valve anatomy and function and evolving surgical and interventional procedures available to address tricuspid regurgitation (TR).

The majority of TR is functional in nature, as opposed to that caused by primary diseases of the valve. It was previously believed that TR secondary to left sided valve disease would resolve after correction of the underlying pathology. This is now known not to be true, and in fact annular dilatation, TR and RV dysfunction may continue to progress after surgery of the aortic or mitral valve (2-4). An area of ongoing controversy is whether TR warrants 
operative attention at the time of concurrent $M V$ surgery and if so, at which grade of severity and whether any specific valve characteristics should be utilized in making this decision (5).

This article aims to give an overview of the echocardiographic assessment of the TV, parameters available to grade the severity of TR and how these may be utilized to assist the surgeon considering intervention.

\section{Anatomy of tricuspid valve relevant to the echocardiographer}

The TV apparatus, similar to that of the other atrioventricular valve consists of leaflets, chordae tendinae, papillary muscles, an annulus and myocardium of the RA and RV. There is very little fibrous tissue contributing to the annulus, leading to dynamic motion during the cardiac cycle and a susceptibility to dilatation.

The TV is the largest and most apically positioned of the valves with an annular area of $8-12 \mathrm{~cm}^{2}$ (6). It has three leaflets; anterior, posterior and septal with the anterior leaflet being the largest.

The geometry of the leaflets, chordae tendineae and papillary muscles is highly variable. The septal leaflet is the most consistent, the others being more variable, often with several scallops and multiple commissures (7). Three papillary muscles usually exist, however these too vary in size and number, the posterior at times being absent (8).

Similar to the mitral valve, the annulus of the tricuspid has a non-planar saddle shape, with high and low points. As the small septal leaflet is fairly fixed to the underlying septum, dilation is thought to occur primarily in the septal-lateral dimensions. This dilatation results in a more circular and planar shaped annulus, contributing to the pathophysiological mechanism of functional TR $(9,10)$.

\section{Pathology involving the TV}

The TV can be affected by many conditions, however the most prevalent, and thus of most relevance to the echocardiographer is regurgitation.

\section{Tricuspid regurgitation}

The most recent American Heart Association guidelines classifies regurgitation of the TV into that caused by either primary or functional mechanisms.

"Primary" regurgitation relates to defects of the leaflets themselves. This includes rheumatic, myxomatous and carcinoid disease. Ebstein's anomaly, endocarditis, trauma and iatrogenic conditions including pacemaker-lead induced leaflet changes (9) are other processes that may be responsible for primary TR.

"Functional" TR is by far the most prevalent pathological mechanism, responsible for $75 \%$ of TR. Causes include left heart disease (dysfunction/valve disease) resulting in pulmonary hypertension, other causes of pulmonary hypertension, and any cause of RV (myocardial disease, RV ischemia/infarction, pulmonary embolism) or right atrial (atrial fibrillation) dilatation (9). The most common cause of functional TR encountered during cardiac surgery is that due to valve disease and dysfunction of the left side of the heart.

Small degrees (trace to mild) of TR are common with a population prevalence of up to $65-75 \%$ and may even be referred to as "physiological" TR. Echocardiography of such TR demonstrates normal leaflets, no dilatation of the $\mathrm{RV}$ and a thin central jet localized to a small region adjacent to the valve which may not persist throughout systole (8).

\section{Iatrogenic tricuspid regurgitation}

The ever-increasing prevalence of devices placed across the TV, such as pacemaker or defibrillator wires can result in a degree of iatrogenic TR. Related complications such as infective endocarditis may result in significant leaflet destruction and more severe TR. Very rarely, complications related to these devices may result in tricuspid stenosis (TS) (Figures 1,2).

\section{Tricuspid regurgitation from myocardial pacing}

Asynchronous RV pacing may precipitate TR, with subsequent resolution upon cessation of pacing (11). This should be considered when new or worsened TR is observed post $\mathrm{CPB}$ as ventricular pacing may be contributing.

\section{Tricuspid stenosis}

As the TV is the largest of the cardiac valves, significant TS is uncommon. Causes include rheumatic and carcinoid heart disease, endocarditis, obstruction by tumors or rarely congenital malformations (1).

\section{Congenital diseases}

Some congenital heart disease (CHD) may lead to dysfunction of the TV. These include Ebstein's anomaly, 


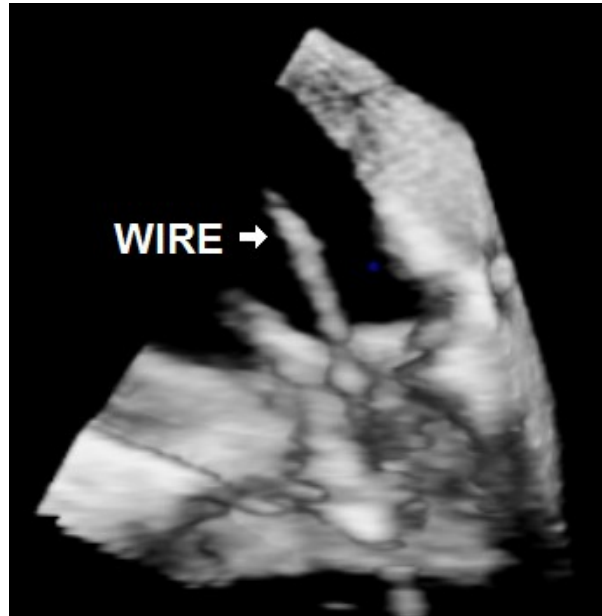

Figure $13 \mathrm{D}$ transesophageal echocardiography of wire across the tricuspid valve.

tricuspid atresia and other atrioventricular abnormalities.

Ebstein's anomaly may lead to significant TR. The key findings are a large anterior leaflet and apical displacement of the TV into the RV with "atrialization" of the remaining ventricle (12). Tricuspid atresia is a rare congenital cardiac abnormality that results in absence of the TV. This condition often occurs with hypoplasia of the RV, an ASD and a VSD (13).

\section{Transthoracic echocardiography (TTE) assessment}

The most commonly performed assessment of the TV is with transthoracic (TTE) imaging. The benefits of this modality include: it is non-invasive, can be repeated with relative ease and is comfortable and low risk for patients.

The TTE examination utilizes four main imaging window: the parasternal, subcostal, apical and suprasternal. Within these, the main views for visualization of the TV are the parasternal LAX, apical four chamber and subcostal views, however none of these windows can image all leaflets of the TV simultaneously (8). The current AHA/ACC guideline defines the "tricuspid annular dilation", at which TV repair should be considered as $>40 \mathrm{~mm}$ or $>21 \mathrm{~mm} / \mathrm{m}^{2}$, and is based on a TTE 4 chamber measure (14).

These 4 windows are also utilized for 3D TTE imaging, which conversely, is able to show all the three TV leaflets simultaneously (15). 3D TTE offers detailed views of TV structures in the majority of patients, however it is still reliant upon adequate 2D images (16) (Figures 3,4).

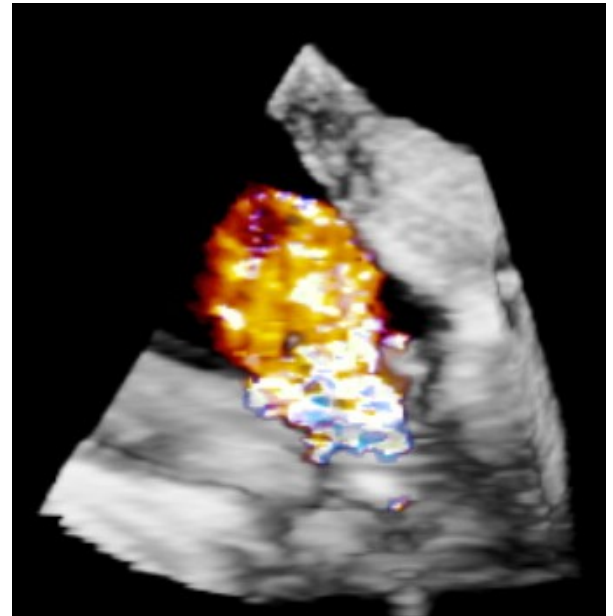

Figure $23 \mathrm{D}$ of tricuspid regurgitation, in part from the implantable cardioverter-defibrillator wire.

TTE is important in assessing the severity of TR and RVSP as the images are obtained in a conscious patient with normal resting hemodynamics. A baseline TTE study is also used to elucidate the etiology of TR, measure the size of right-sided chambers and the IVC, assess RV systolic function, and characterize any associated left sided disease (14).

TTE can quantifying the effects of spontaneous respiration on hemodynamic measures across the TV and on other structures, such as the IVC. These measures can assess the fluid status and volume responsiveness of the patient, as well as estimating values such as the CVP. Performing Doppler echocardiographic measurements across the TV, and looking for variation during spontaneous respiration, can be used in investigating constrictive pericarditis, tamponade and other conditions of the pericardium (17).

A technically adequate and complete TTE examination may be difficult to perform in some patients. These include the obese, those with hyperinflated lungs (such as COPD), intubated patients, those who cannot be optimally positioned and post cardiac surgical patients.

\section{Transesophageal echocardiography (TEE) modalities}

Although most patients presenting for cardiac surgery will already have had a preoperative TTE, a comprehensive intraoperative 2D TEE examination remains important. Such a study can investigate for additional findings that 


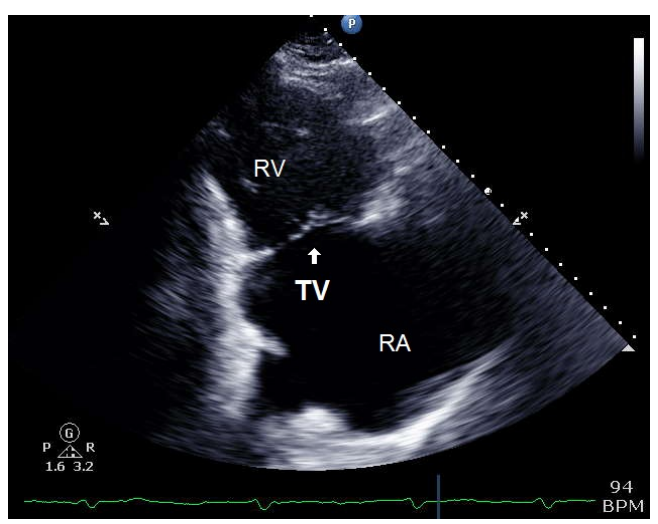

Figure 3 Transesophageal echocardiography of right ventricular (RV) inflow (from parasternal long-axis view). RA, right atrium; TV, tricuspid valve.

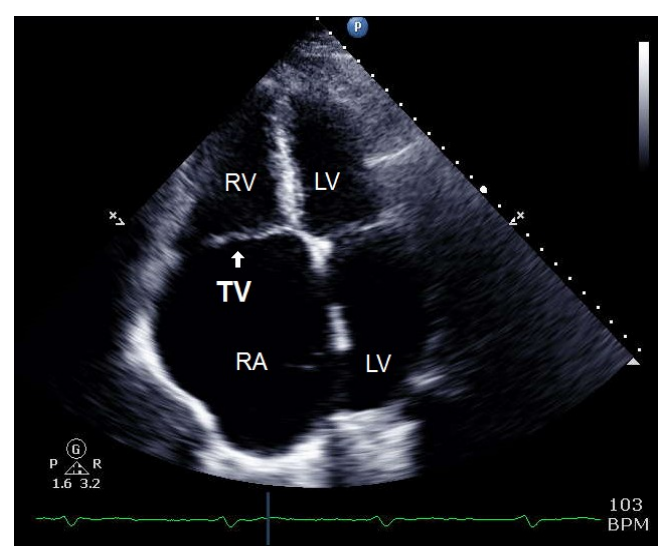

Figure 4 Transesophageal echocardiography apical 4 chamber view. LA, left atrium; LV, left ventricle; RA, right atrium; RV, right ventricle; TV, tricuspid valve.

may have been missed on TTE and can assist in operative planning and surgical decision making.

The altered loading conditions of general anesthesia will change the appearance of TR, generally making it appear less severe. The favorable location of the TEE transducer, close to the heart, allows for higher resolution imaging than with TTE with improved delineation of anatomical structures.

Once inserted, the TEE probe has four standard transducer positions, and associated imaging planes, within the esophagus and stomach:

* Upper esophageal (UE): this imaging plane is used to image the ascending aorta and related great vessels including the pulmonary veins;

\begin{tabular}{|l}
\hline Table 1 Absolute and relative contraindication \\
\hline Absolute contraindications \\
\hline Perforated viscus \\
\hline Esophageal stricture \\
\hline Esophageal tumor \\
\hline Esophageal perforation, laceration \\
\hline Esophageal diverticulum \\
\hline Active upper Gl bleed \\
\hline Relative contraindications \\
\hline History of radiation to neck and mediastinum \\
\hline History of Gl surgery \\
\hline Recent upper Gl bleed \\
\hline Barrett's esophagus \\
\hline History of dysphagia \\
\hline Restriction of neck mobility (severe cervical arthritis, atlantoaxial \\
joint disease) \\
\hline Symptomatic hiatal hernia \\
\hline Esophageal varices \\
\hline Coagulopathy, thrombocytopenia \\
\hline Active esophagitis \\
\hline Active peptic ulcer disease \\
\hline
\end{tabular}

* Mid esophageal (ME): this plane offers the best views of the 4 chambers of the heart. The majority of TEE images are obtained within this plane;

* Transgastric (TG): deeper than the ME plane, within the stomach. It is here that the familiar circular cross sectional views of the $L V$ and $R V$ are obtained;

* Deep TG: deeper again than the TG views. This view is used primarily for aligning the aortic valve for Doppler interrogation.

Although often considered a reasonably "non-invasive" instrument, TEE comes with its own unique risks. There are a number of absolute and relative contraindications to TEE utilization (5) (Table 1). The overall rate of complications from TEE placement and intraoperative usage is $0.2 \%$ (18) (Table 2).

\section{Basic imaging principles}

A systematic approach to examining the TV by 


\begin{tabular}{|ll}
\hline Table 2 Incidence of complications & \\
\hline Complications & Incidence (\%) \\
\hline Mortality & 0 \\
\hline Major morbidity & $0-1.2$ \\
\hline Major bleeding & $0.03-0.8$ \\
\hline Esophageal perforation & $0-0.3$ \\
\hline Minor pharyngeal bleeding & 0.01 \\
\hline Severe odynophagia & 0.1 \\
\hline Dental injury and ETT malposition & Both 0.035 \\
\hline
\end{tabular}

echocardiography should be utilized. This involves evaluating the size of the right-sided cardiac chambers, motion of the interventricular septum and obtaining Doppler parameters and color imaging, in at least 2 orthogonal planes wherever possible. The size of the IVC and response to respiration (if possible) as well as hepatic vein flow pattern helps evaluate right atrial pressure and adaptations to volume overload (19).

The AHA/ACC guidelines for assessment of TR utilize central jet area, continuous wave Doppler (CWD) jet density and contour, hepatic vein flow and the hemodynamic consequences of TR on RV/RA/IVC size and RV function (14). The American Society of Echocardiography (ASE) and the European Association of Echocardiography (EAE) both recommend very similar parameters (A discussion of the benefits/limitations of these measures from the EAE is included as Table S1).

\section{D imaging}

The TV apparatus, and all leaflets of the TV should be carefully examined in a number of views to assess their morphology and function as well as to look for any pathological processes that may be causing primary TR. The RV should also be assessed thoroughly during all phases of the cardiac cycle, examining for potential dilatation and/or dysfunction leading to secondary TR. The size of the RA and IVC should also be measured.

The recent ASE guidelines for performing a comprehensive intraoperative TEE lists 28 "standard" views suggested as the minimum imaging set to be obtained (5). This is an increase from the 20 standard views in the previous guidelines from 2000. At least eight of these views allow for imaging of the TV, and some have been included

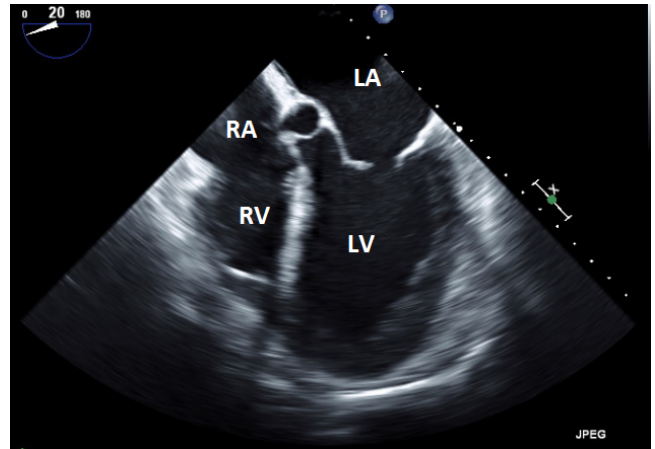

Figure 5 Mid-esophageal 5 chamber view; LA, left atrium; LV, left ventricle; RA, right atrium; RV, right ventricle.

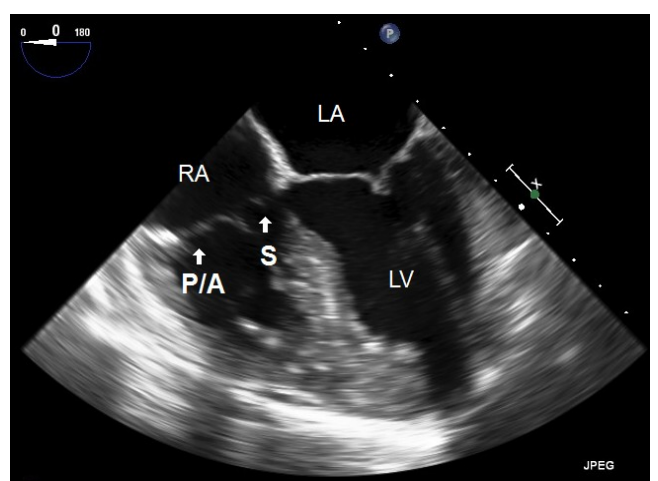

Figure 6 Mid-esophageal four chamber view. LA, left atrium; LV, left ventricle; P/A, posterior/anterior; RA, right atrium; S, septal.

specifically for their improved imaging of the right side of the heart.

* ME five chamber (ME5C) (Figure 5):

- This is the first of the standard images obtained in the ME plane. The " $5^{\text {th }}$ chamber" in this view is the LVOT;

- As this is "higher" than the ME4C view, the TV leaflets imaged are likely to be the septal and anterior.

* ME four chamber (ME4C) (Figure 6):

- This is one of the most frequently utilized and familiar TEE views and offers excellent imaging of the four chambers of the heart;

- In this view the septal leaflet is closest to the interventricular septum and either the anterior or posterior leaflet is viewed on the left of the image;

- It is deeper than the ME5C and as such the AV and LVOT should not be seen.

- ME RV inflow outflow (Figure 7):

- This view, as the name suggests offers a view of the 


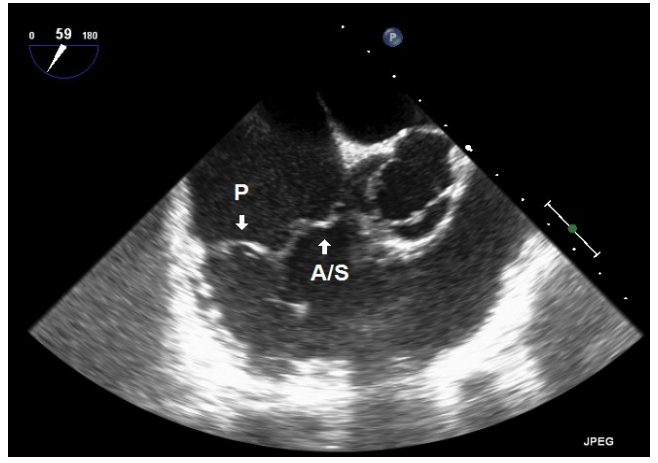

Figure 7 Mid-esophageal right inflow view. P/A, posterior/ anterior; S, septal.

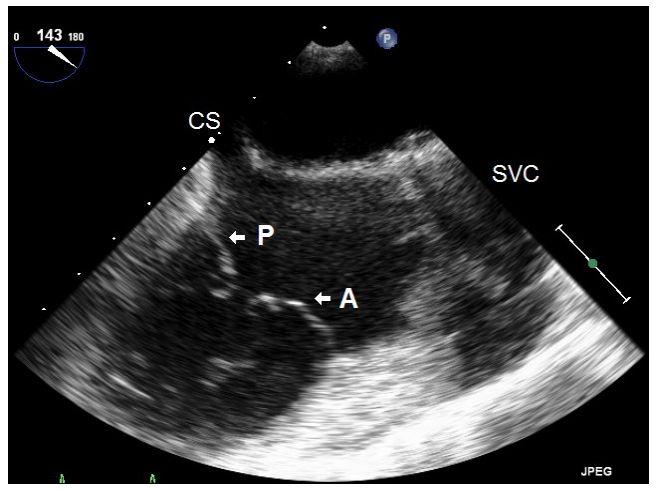

Figure 8 Mid-esophageal modified bicaval view. A, anterior; CS, coronary sinus; $\mathrm{P}$, posterior; SVC, superior vena cava.

RA, RV, RVOT and PA as well as the function of the RV free wall;

- Both the TV and the pulmonic valve are imaged in this view;

- the anterior or septal leaflets are closest to the AV, and the posterior to the left of screen.

* ME modified bicaval (Figure 8):

- This is similar to the standard "ME bicaval view", with the IVC to the right of the screen and the SVC to the left, however is focused more on the TV;

- This "modification" allows for better alignment of the TR jet with the Doppler signal;

- The posterior leaflet is to the left of screen and the anterior to the right.

\section{Transgastric views}

Some of these views are new from the previous guidelines

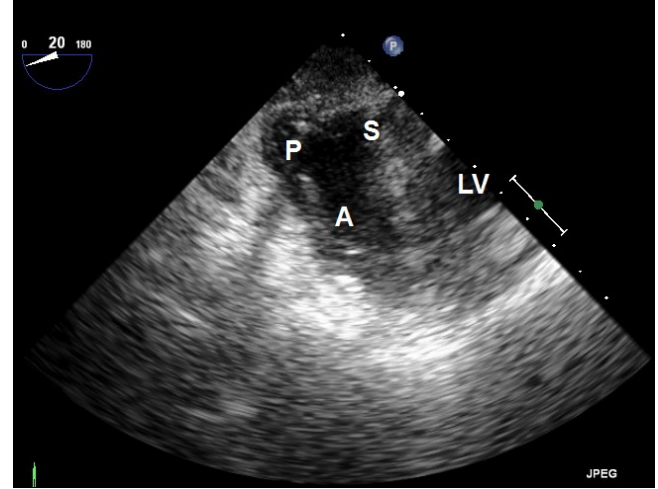

Figure 9 Transgastric short axis view; A, anterior; LV, left ventricle; $\mathrm{P}$, posterior; $\mathrm{S}$, septal.

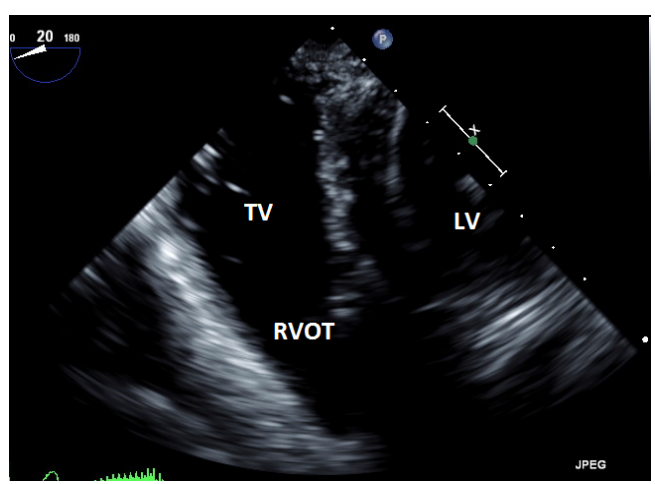

Figure 10 Transgastric right ventricle basal view. $\mathrm{LV}$, left ventricle; RVOT, right ventricular outflow tract; TV, tricuspid valve.

and offer improved imaging of the TV and the related apparatus. These include:

* TG Basal SAX (Figure 9):

- Although primarily focused on the basal LV and the mitral valve, the transducer can be positioned to focus more on the TV and basal RV in short axis;

- The septal leaflet is near the LV, whilst the posterior and anterior leaflets are in the near and far field respectively.

* TG RV Basal view (Figure 10):

- Allows imaging of the TV, the RVOT as well as the PV.

* TG RV inflow (Figure 11):

- The anterior and inferior walls of the RV are imaged in addition to the papillary muscles, chordae and TV;

- The posterior leaflet is usually imaged in near field. 


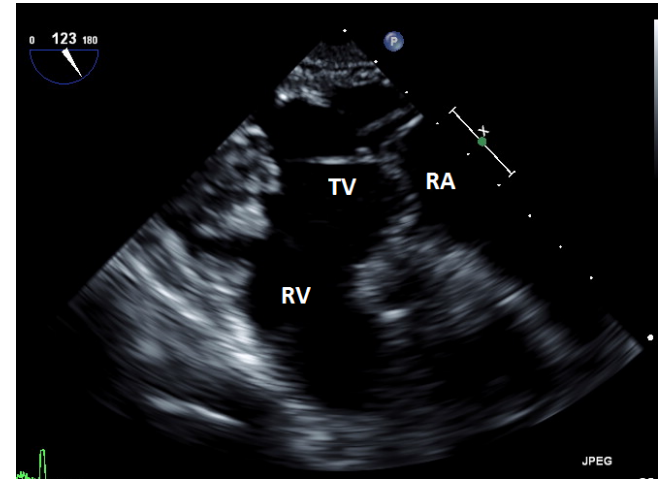

Figure 11 Transgastric right ventricular (RV) inflow view. RA, right atrium; TV, tricuspid valve.

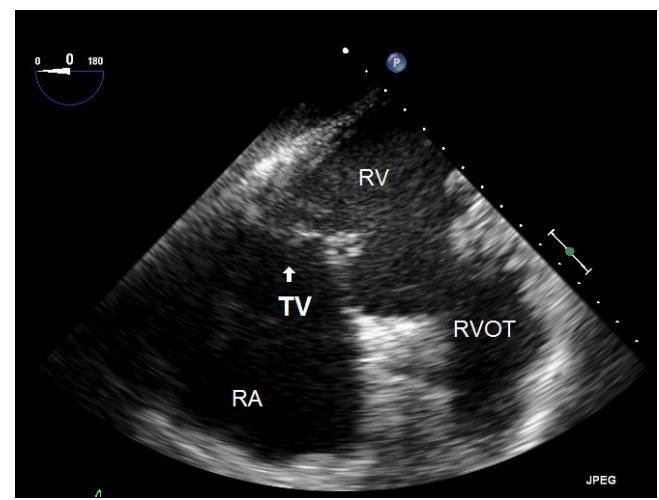

Figure 12 Transgastric right ventricle (RV) inflow-outflow. RA, right atrium; RVOT, right ventricular outflow tract; TV, tricuspid valve.

* TG RV inflow-outflow (Figure 12):

- As the name suggests this TG image offers view of the RA, RV, RVOT and PA;

- Typically in this view the posterior leaflet usually in near field and the anterior in the far field.

Specific TEE views of the right heart have previously been suggested that ensured visualization of the TV in two or more planes in $97 \%$ of the patients investigated, increasing the likelihood of a full examination including eccentric jets (20). Some of these views, such as the TG RV Basal SAX and TG RV Inflow-Outflow views have subsequently been incorporated into the more recent ASE guideline. An additional "right heart" specific view that offers a unique perspective on the TV is:

- Deep TG RV inflow-outflow (Figures 13,14):

- The Deep TG TEE view is most commonly used to align the AV and LVOT with the Doppler beam,

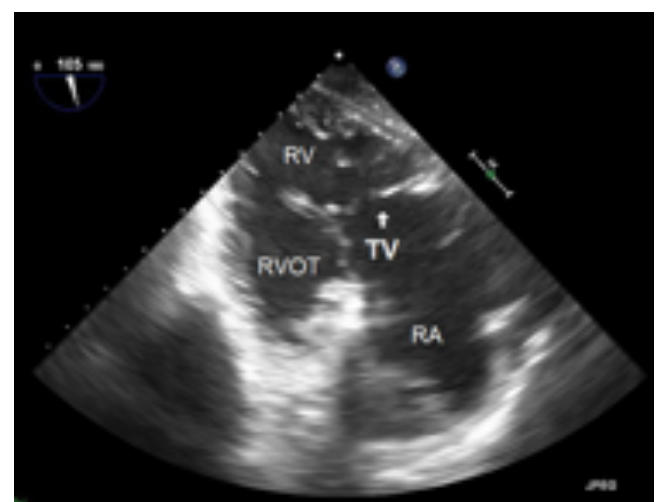

Figure 13 Deep transgastric right ventricle (RV) inflow-outflow. RA, right atrium; RVOT, right ventricular outflow tract; TV, tricuspid valve.

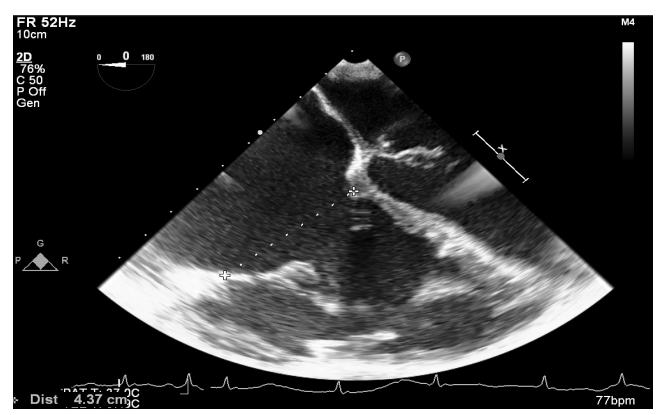

Figure 14 Mid-esophageal four chamber view, with annulus enlarged at $44 \mathrm{~mm}$.

allowing for measuring velocities and gradients across the AV and within the LVOT;

- With a small modification it offers an additional view of the RV and related structures.

The normal adult TV annular diameter is considered to be $28 \pm 5 \mathrm{~mm}$ on TEE assessment when viewed in the ME4C view. There is a linear relationship between annular diameter and TR volume, with a diastolic diameter of $>40 \mathrm{~mm}\left(>21 \mathrm{~mm} / \mathrm{m}^{2}\right)$ representing significant dilation (14). A systolic diameter $>32 \mathrm{~mm}$ or a diastolic diameter $>34 \mathrm{~mm}$ are measurements in favor of more significant TR (8). Of note the normal contraction of the TV annulus can result in a variation of annular area up to $25 \%$ during the cardiac cycle $(8,9)$.

\section{TR: color flow Doppler (CFD)}

CFD can be used to rapidly and easily identify the presence or absence of TR. Despite this, it should not be used as 


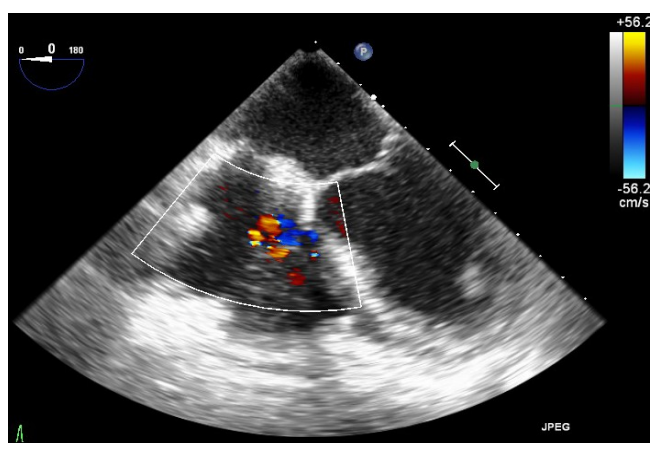

Figure 15 Trace tricuspid regurgitation on color flow Doppler.

the sole parameter for quantifying anything more than a small degree of TR due to its significant dependence on hemodynamic and technical factors (8). As a general rule, jets that extend deep into the RA represent more TR than small central jets that appear just superior to the leaflets.

The simplest approach to the initial evaluation of TR severity is to utilize CFD imaging in several views to establish the characteristics, direction and size of any regurgitant jet. A centrally directed jet may appear more significant than a wall hugging jet, but this can be misleading (19). The CFD approach may also be unreliable in the setting of leaflet malcoaptation, which results in a very large regurgitant orifice, across which flow acceleration is minimal (21) (Figures 15,16).

\section{TR: spectral Doppler analysis}

Doppler ultrasound measures the velocities of red blood cells and uses these velocity characteristics to calculate the hemodynamic qualities of blood flow. Pulse wave Doppler (PWD) measures lower velocities from a specific location of interest (range specificity) whilst CWD measures all velocities along the plane of interrogation, but cannot localize the source of the identified velocities (range ambiguity).

Doppler echocardiography utilizes the modified Bernoulli equation to calculate the pressure characteristics from measured velocities. In Doppler analysis it is important that the interrogation axis is as parallel as possible to the regurgitant jet under investigation as misalignment greater than 30 degrees can introduce significant measurements errors.

\section{Pulse wave Doppler}

In the absence of TS, peak velocity of the early diastolic

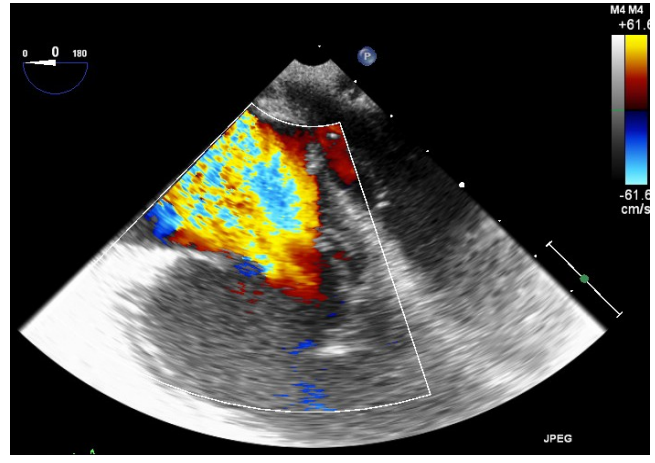

Figure 16 Severe tricuspid regurgitation on color flow Doppler.

filling (E velocity) increases in proportion to the degree of TR. Tracings are obtained at the tricuspid leaflet tips with a peak E velocity $>1 \mathrm{~m} / \mathrm{s}$ suggesting severe TR (8).

\section{Continuous wave Doppler}

CWD interrogation of the TV can be used in the presence of TR to estimate the RVSP. Not only are the velocities obtained useful, but also the shape and appearance of the CWD imaging is of relevance. A dense CWD signal with a full envelope indicates more severe TR than a faint signal (Figures 17,18).

\section{More quantitative measures of TR severity}

As described, both the CFD measures and Doppler analysis of TR are susceptible to error. Increasingly, quantitative echocardiographic measures are necessary to grade TR severity and assist in decision making. The vena contracta (VC), proximal isovolumetric surface area (PISA) and effective regurgitant orifice area (EROA) techniques can all be utilized. Improved ultrasonographic technology is making obtaining these advanced measures easier.

\section{Vena contracta}

The VC measures the narrowest portion of the regurgitant jet as it passes through the valve orifice. Accuracy of this measure relies on ensuring standardized echocardiographic settings and observing the valve in multiple planes to ensure the most appropriate jet is obtained. A VC greater or equal than $7 \mathrm{~mm}$ favors severe, whereas $<6 \mathrm{~mm}$ may be considered either mild or moderate TR. Intermediate 


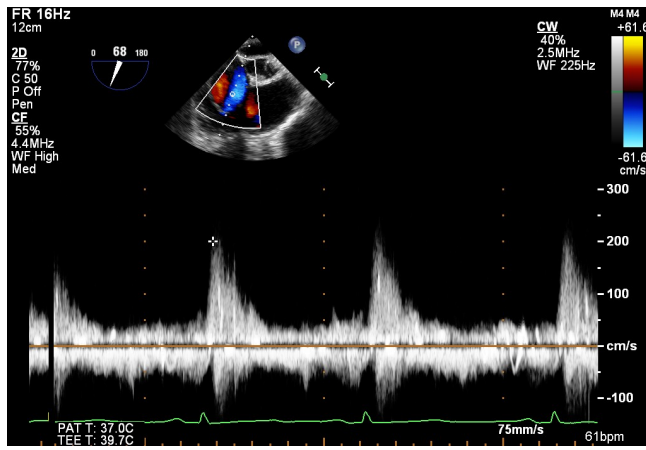

Figure 17 Mild tricuspid regurgitation on continuous wave Doppler.

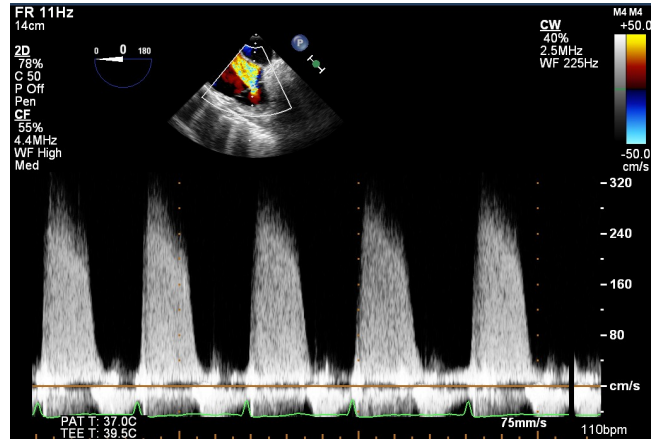

Figure 18 Severe tricuspid regurgitation on continuous wave Doppler.

Table 3 ASE stages of TR [Modified from (19)]

\begin{tabular}{llll}
\hline Parameter & Mild & Moderate & Severe \\
\hline Tricuspid valve & Usually normal & Normal or abnormal & Abnormal/flail/poor coaptation \\
\hline RA/RV/IVC size & Normal & Normal or dilated & Usually dilated \\
\hline Jet area-central jets $\left(\mathrm{cm}^{2}\right)$ & $<5$ & $5-10$ & $>10$ \\
VC width $(\mathrm{cm})$ & Not defined & Not defined, but $<0.7$ & $>0.7$ \\
PISA radius $(\mathrm{cm})$ & $\leq 0.5$ & $0.6-0.9$ & $>0.9$ \\
CW jet density and contour & Soft and parabolic & Dense, variable contour & Dense, triangular; early peaking \\
Hepatic vein flow & Systolic dominance & Systolic blunting & Systolic reversal \\
\hline
\end{tabular}

ASE, American Society of Echocardiography; PISA, proximal isovolumetric surface area. CW, continuous wave; IVC, inferior vena cava; RA, right atrium; RV, right ventricle; $\mathrm{VC}$, vena contracta.

values are not accurate at distinguishing mild from moderate TR (19).

The assessment of $\mathrm{VC}$ by $3 \mathrm{D}$ echo, which aims to resolves geometric assumptions related to the shape of the regurgitant orifice are still reserved largely for research purposes (8).

\section{Proximal isovolumetric surface area}

PISA is commonly utilized in the assessment of mitral regurgitation, but may be used in an analogous manner when quantifying TR. Blood flow accelerates as it approaches the regurgitant orifice, resulting in concentric shells of increasing velocity, depicted with color Doppler. The area of these hemispheric shells is the PISA. The radius $(\mathrm{mm})$ of the hemisphere from the narrowest section of the regurgitant jet is a measure of severity. Mild TR $<5 \mathrm{~mm}$, Moderate TR 6-9 $\mathrm{mm}$ and $>9 \mathrm{~mm}$ is severe TR.
(Table 3). PISA may be cumbersome to perform and can underestimate the severity of TR (8).

\section{EROA/regurgitant volume}

The most quantitative assessment of valvular regurgitation are measures of the regurgitant jet orifice area and regurgitant volumes. Quantification of TR in this manner has been validated, however is rarely performed intraoperatively because of its complexity as well as time constraints (22). These volume measures are also infrequently used in clinical practice, partially due to their complexity and partially because of their vulnerability to measurement errors (19). An EROA $>40 \mathrm{~mm}^{2}$ or a Regurgitant Vol of $>45 \mathrm{~mL}$ indicates severe TR (8).

$3 \mathrm{D}$ echocardiography may reduce some of the geometric assumptions of 2D techniques, potentially making intraoperative utilization of these quantitative 


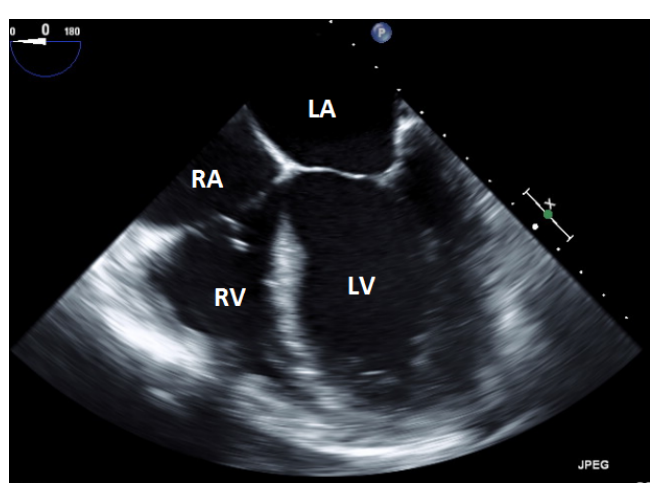

Figure 19 Normal RV dimensions.

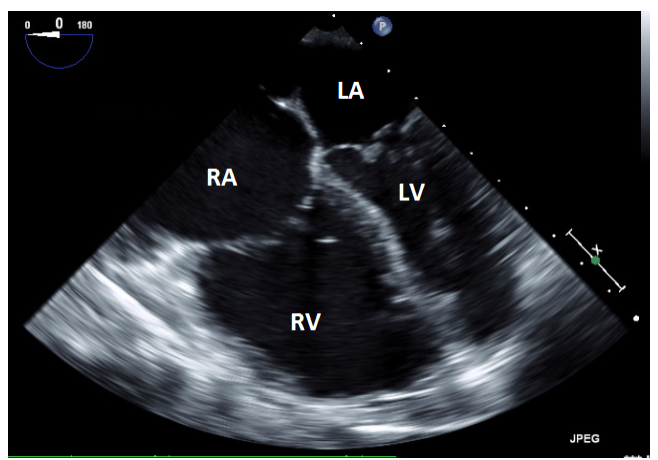

Figure 20 Abnormal RV dimensions.

approaches more practical and accurate. With $3 \mathrm{D}$ echocardiography, regurgitant volume $>75 \mathrm{~mL}$ is likely to indicate severe TR (8).

\section{Additional echo measures in patients with TR}

In a patient with anything more than mild TR, evaluation of RA volume, the function and dimensions of the RV and an estimation of the RVSP are mandatory. (8) Findings such as enlargement of the RA and RV, and diastolic interventricular septal flattening often accompany significant chronic TR (19).

The RA can be well imaged in a number of the previously described TEE views. The $2 \mathrm{D}$ area of the RA is relatively easily measured, however accurate assessments of RA volume are more difficult to perform (23).

The simplest approach to assessing RV dilatation is by measurement of the RV cavity end-diastolic diameters. This is performed using both horizontal and vertical RV measures in the ME4C view (Figures 19,20).

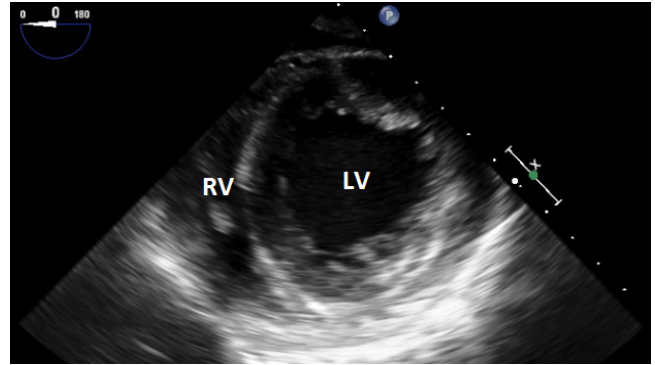

Figure 21 Normal right ventricle (RV) on short axis view.

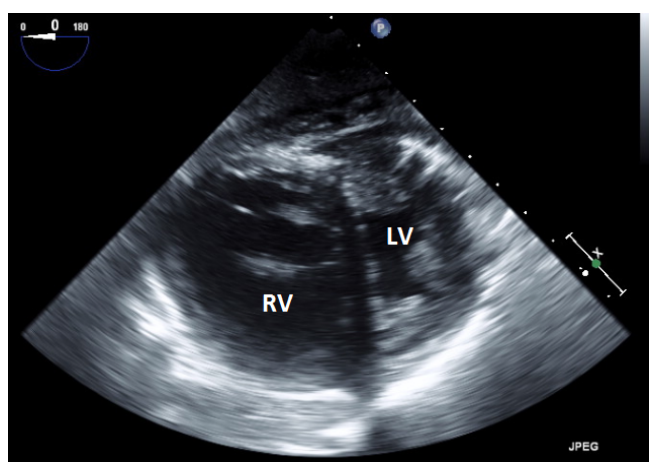

Figure 22 Abnormal right ventricle (RV) on short axis view.

Further assessment of the RV dimensions and function can be performed in the TG SAX views. In these views it is possible to assess the size, thickness and function of the RV. Timing of the imaging is important, as is measurement of the interventricular septal motion. RV pressure overload results in a hypertrophied $R V$ that assumes a circular shape at end-systole. RV dilatation from volume overload results in a "D" shaped (flattened) $\mathrm{LV}$ at the period of maximal filling, that is, end-diastole (Figures 21,22).

Another qualitative assessment of RV size and function can be obtained by observing how much of the "apex" of the heart appears to be made up by the RV. Ordinarily this should be none, the apex being made entirely of the LV. As such, when the RV is "apex forming", in images such as a ME4C view, this suggests RV dilatation.

PWD of the hepatic veins can corroborate the assessment of TR severity. With increasing severity of TR, the normally dominant systolic wave is blunted, and with severe TR, systolic flow reversal occurs. Although there are other factors which can influence this appearance, the sensitivity for severe TR is high (80\%) (19). The anterior hepatic vein 


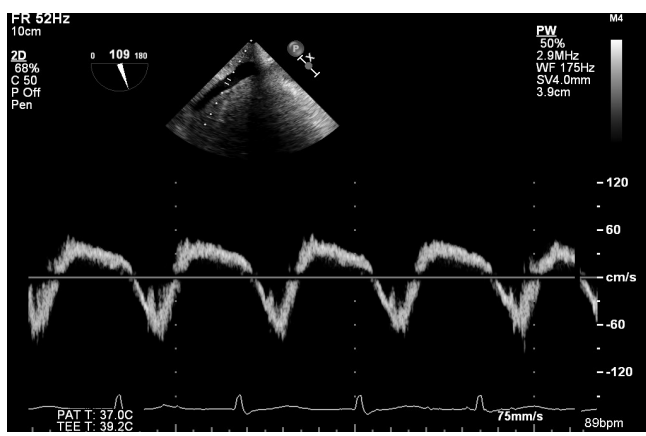

Figure 23 Hepatic vein systolic flow reversal.

is the best structure in which to obtain a suitable on-axis view (Figures 23,24).

\section{Additional measures in functional TR}

With tricuspid annular dilatation, the loss of leaflet coaptation and increased tethering of the leaflets and subvalvular apparatus are important factors in the development of functional TR.

As with MR, echocardiographic measures of these pathophysiological processes can be made as part of quantifying the severity of regurgitation. Tethering can be evaluated by measurement of the tethering distance (distance between the tricuspid annular plane and the coaptation point) and the systolic tenting area (area between the annulus and the tricuspid leaflets) in mid-systole (24). A tenting area $>1.6 \mathrm{~cm}^{2}$ has been shown to be associated with severe TR, while a tethering distance of $>8 \mathrm{~mm}$ is significant (25).

\section{Assessment of TS}

Although a relatively uncommon condition, echocardiography can be utilized to assess and quantify the severity of stenotic disease of the TV. Echocardiographic findings suggestive of hemodynamically significant TS include (14):

* Mean pressure gradient $>5 \mathrm{mmHg}$;

* $\mathrm{T}_{1 / 2}>190 \mathrm{~ms}$

* Valve area by continuity $<1 \mathrm{~cm}^{2}$.

\section{Continuous wave Doppler}

CWD interrogation is performed with the line of interrogation through the TV. Tracing any resultant

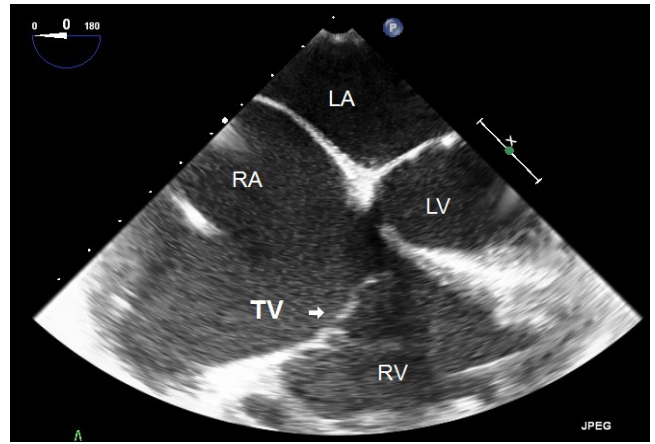

Figure 24 Enlarged right atrium and right ventricle from chronic tricuspid regurgitation.

envelope will measure both the peak and mean pressure gradients.

\section{$T_{1 / 2}$ assessment (pressure half time)}

The pressure half time, commonly used in MS, is the time interval (in milliseconds) between the maximum tricuspid gradient and the time point where the gradient is half the maximum initial value. The decline in the velocity of transtricuspid blood flow is inversely proportional to valve area.

\section{Continuity equation}

Valve area can be determined by using the continuity equation, based on the conservation of mass. The volume of blood passing though one area of the heart (such as the LVOT or RVOT) is equal to the volume of blood through the TV. Using echocardiography, both the area and the VTI (flow velocity, averaged over time) of the LVOT can be determined. By obtaining the VTI of the TV the equation can be solved for TV area.

* Continuity equation: $\mathrm{A}_{\mathrm{AREA}} \times \mathrm{A}_{\mathrm{VTI}}=\mathrm{B}_{\mathrm{AREA}} \times \mathrm{B}_{\mathrm{VTI}}$

$* \mathrm{TV}_{\mathrm{AREA}}=\mathrm{LVOT}_{\mathrm{AREA}} \times \mathrm{LVOT}_{\mathrm{VTI}} / \mathrm{TV}_{\mathrm{VTI}}$

The continuity equation is difficult to use in TR due to the prevalence of mixed valve disease. Although performing $3 \mathrm{D}$ quantification of the orifice area is possible it is not sufficiently validated enough to be recommended (26).

\section{Issues with echo measurement}

When considering the echocardiographic assessment of the $\mathrm{TV}$, perhaps one of the most important aspects is to ensure that the TV is in fact examined. In routine clinical practice it is surprising how often the TV is inadequately imaged. 


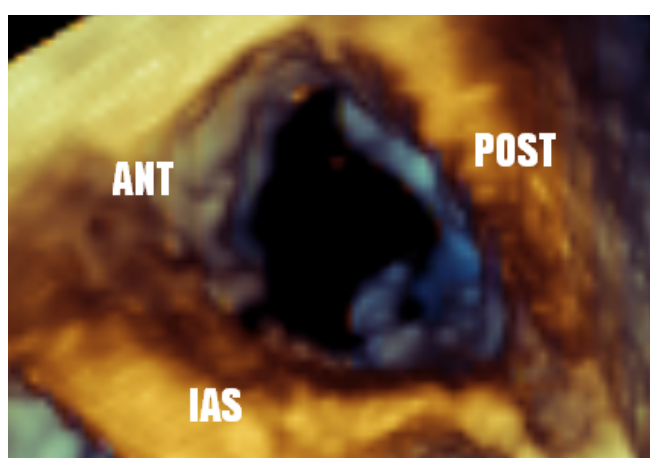

Figure $253 \mathrm{D}$ imaging of the tricuspid valve (right atrial view). Ant, anterior; IAS, interatrial septum; post, posterior.

A thorough and systematic examination of the TV in a number of views is an integral part of the comprehensive TEE examination, as outlined by the ASE guidelines, and should always be obtained whenever possible.

Central to the practice of echocardiography is an understanding that single $2 \mathrm{D}$ image slices can lead to misrepresentation of the 3D structures under investigation. To minimize this potential for errors structures of interest should always be imaged utilizing multiple TEE views, from differing angles and with standard machine settings to avoid artifact.

The appearance of TR by can be significantly influenced by RV preload, afterload, and RV systolic function (9). Thus the severity of TR may be diminished under general anesthesia when compared to a TTE performed in a resting conscious state. Inter-observer agreement can be difficult to achieve. The use of an algorithm may improve the agreement of severe/non-severe TR between observers (27).

In a small study by Maslow and colleagues they compared 4 TEE views (ME4C, ME RV inflow/outflow, ME RV inflow/coronary sinus window and TG RV inflow/outflow) with direct intraoperative measures of the TV annulus in an arrested heart. The TEE view in which the annular measure obtained demonstrated best agreement with the measure obtained by the surgeon was the TG RV inflow/outflow view at end diastole (or greatest diameter) (28).

Occasionally, despite detailed echocardiographic assessment, there may still be uncertainty to the degree of TR present in a particular patient. In these situations a number of echocardiographic parameters should be obtained in multiple views. Not all measures may indicate the same severity of TR, however it should be possible to reach a consensus on the likely grade. Furthermore, other

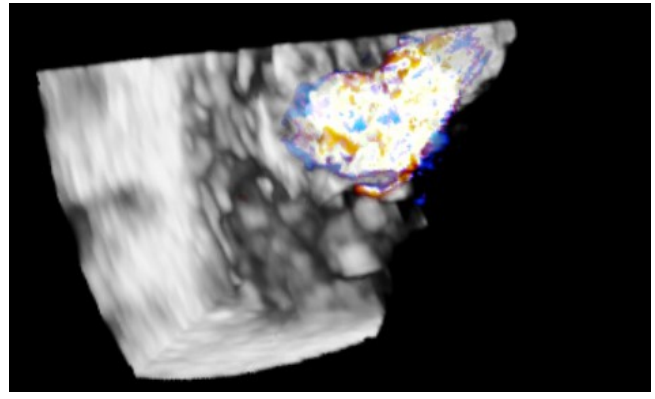

Figure $263 \mathrm{D}$ color of large central tricuspid regurgitation jet.

parameters influenced by long term TR, such as RA and RV size and annular measurements can also be utilized in determining the likely degree of severity.

\section{D analysis}

As in all other areas of echocardiography, 3D technology is increasingly commonly performed in the assessment of the $\mathrm{TV}$ and the RV. This modality requires specialized probes and advanced image processing. 3D imaging has been particularly helpful in delineating the complex, non-planar, structure of the TV annulus and is offering new insights into the pathophysiology and disease of the TV $(15,29,30)$.

Guidelines for performing and displaying 3D TEE images recommend a standard approached for displaying the en face view of the TV. The image can be displayed as if viewing the TV from either the RA or RV orientation. In both, it is suggested the septal leaflet be displayed at the six o'clock (inferior) position. En face views may be especially helpful in localizing leaflet disease such as prolapse, perforation, or vegetations, as well as localizing the origin of regurgitant jets, or performing planimetry of the tricuspid orifice area (5) (Figures 25,26).

Whilst 3D imaging allows the echocardiographer to display leaflets and valve morphology in a manner that is familiar to surgeons, the greatest benefit is likely to be from quantification programs. Manipulation of $3 \mathrm{D}$ data sets allows for measurements and quantification to be performed in any axis. These manipulations do take time to perform and represent an additional skill to be obtained with added complexity exceeding standard intra-operative TEE.

$3 \mathrm{D}$ echocardiography allows for more accurate measures of parameters such as tricuspid annular dilatation, VC, PISA and EROA without many of the errors or geometric assumptions that $2 \mathrm{D}$ imaging is susceptible to (31). For example, measures of tricuspid annular diameter obtained 


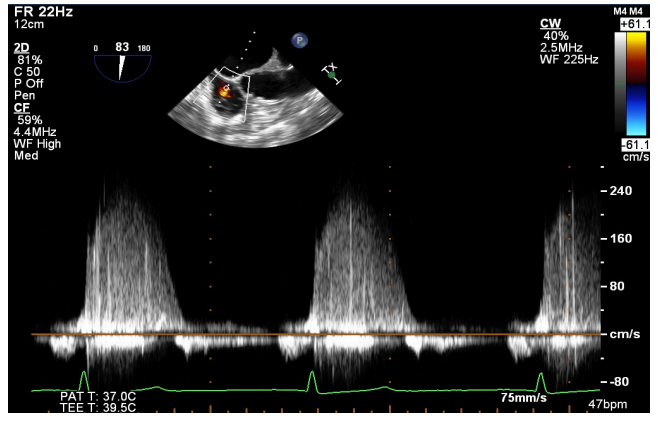

Figure 27 Peak continuous wave Doppler tricuspid regurgitation jet velocity.

by 3D TTE better correlate with those obtained from MRI, whereas 2D TTE consistently underestimate it (32).

3D echocardiography can also be utilized to perform volumetric assessment of ventricular function. Whilst reasonably common for the $\mathrm{LV}$, this technique is less commonly performed in the RV. This has been limited by the complex geometry and systolic function of the RV. Whilst progress is being made, its use in clinical practice remains uncommon.

\section{Other measures of interest related to the TV}

Other useful echocardiographic measurements utilize components of the TV apparatus or regurgitant jet.

\section{Right ventricular systolic pressure}

RVSP can be determined from the peak TR jet velocity using CWD, the simplified Bernoulli equation and the estimated RAP:

* Simplified Bernoulli. Pressure $(\mathrm{mmHg})=4 \times \mathrm{V}^{2}(\mathrm{~V}=$ velocity)

* RVSP $=4\left(\mathrm{~V}^{2}\right)+$ RAP $(\mathrm{V}=$ peak velocity in $\mathrm{m} / \mathrm{sec}$ of the TR jet)

As velocity measures are angle dependent it is recommended to gather TR signals from several windows and use the signal with the highest velocity (23). In the absence of a gradient across the PV this value is equal to PASP. Without an adequate jet of TR it is not possible to calculate RVSP using this technique (Figures 27,28).

Tricuspid annular plane systolic excursion (TAPSE)

TAPSE is a commonly utilized single dimension measure

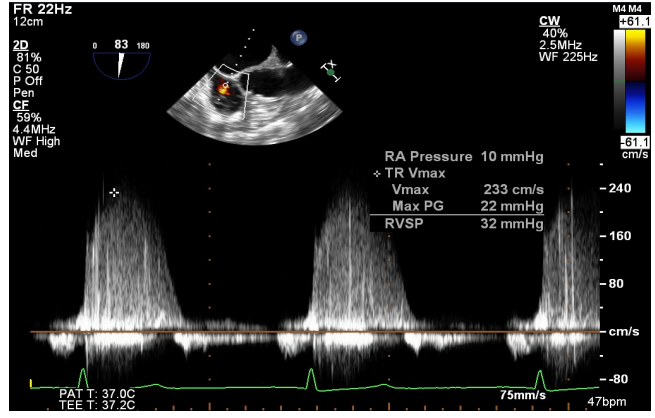

Figure 28 Peak jet velocity estimating PASP. RVSP = 4(V2) + RAP. RASP, right ventricular systolic pressure; RAP, right atrial pressure.

of global RV systolic function. It measures the distance of systolic excursion of the RV annular segment along its longitudinal axis. A TAPSE of $<16 \mathrm{~mm}$ suggests impaired RV systolic function (23). It has a strong evidence base in TTE use, but is less validated for TEE.

\section{RV dP/dT using CWD of TR jet}

This uses the TR CWD signal from 1 to $2 \mathrm{~m} / \mathrm{s}$ and the simplified Bernoulli equation to determine a measure of RV contractility. From 1 to $2 \mathrm{~m} / \mathrm{s}$ represents a $12 \mathrm{mmHg}$ increase in pressure. The $\mathrm{dP} / \mathrm{dT}$ is therefore $12 \mathrm{mmHg}$ divided by the time (in s) needed to obtain this change in velocity. While not recommended for use in normal patients, in patients with $\mathrm{RV}$ dysfunction a value of $<400 \mathrm{mmHg} / \mathrm{s}$ is likely abnormal (23).

\section{Epicardial examination}

Some patients undergoing cardiac surgery may have contraindications to placement of a TEE probe. However, by utilizing an epicardial examination the function of the right ventricle and TV can still be assessed intraoperatively by echocardiography (14).

This technique uses an ultrasound transducer (often a transthoracic probe) within a sterile cover. This can be performed in the same manner as epiaortic scanning of the aorta examining for the presence of plaque and calcification. Many of the conventional imaging modalities can be performed utilizing epicardial imaging.

The epicardial LV LAX and LV basal SAX views allows for evaluation of the TV. Standard 2D imaging can be performed as can Color Doppler and Spectral Doppler investigation of the TV. Assessment of TR with 


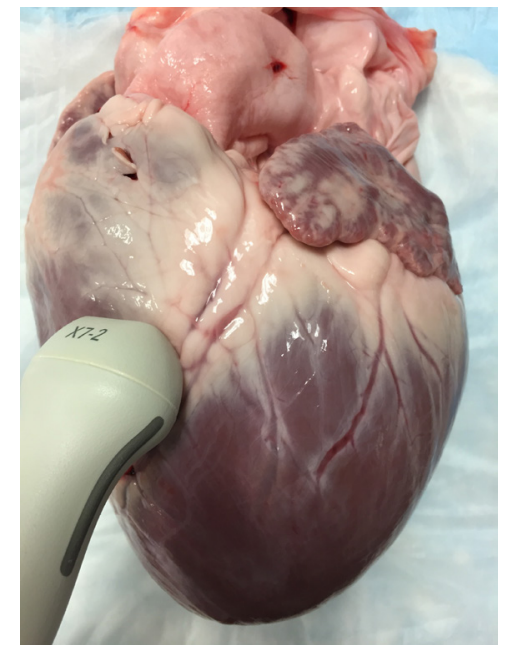

Figure 29 Epicardial basal short-axis view (porcine heart).

an epicardial technique obtains values with reasonable agreement to those from more conventional techniques $(33,34)$ (Figures 29,30).

\section{Other echocardiographic modalities}

Intra cardiac echocardiography (ICE) is increasingly utilized within the practice of interventional cardiology. It involves a single use ultrasound probe inserted via a vascular sheath that emerges in the RA. This initial "home" view demonstrates the TV, RV inflow/outflow and the PV (analogous to the TEE RV inflow-outflow view). ICE offers many of the modalities of conventional echo such as 2D imaging, color and Doppler capabilities, a $360^{\circ}$ rotating transducer and even 3D imaging (35) but with views in a different orientation to those obtained by conventional TEE or TTE.

ICE has some benefits over TEE such as not requiring esophageal placement and the TV can be visualized in many orientations. However it also presents unique issues such as expense, due to the single use probe, the need for large vascular access and it does require additional physician expertise to operate and interpret .

\section{Using TEE to guide decision making-a framework}

In the absence of one "best" measure our approach reiterates the guidance of both the EAE and ASE who advise integrating information from all available parameters to quantify TR. The more congruent the findings regarding

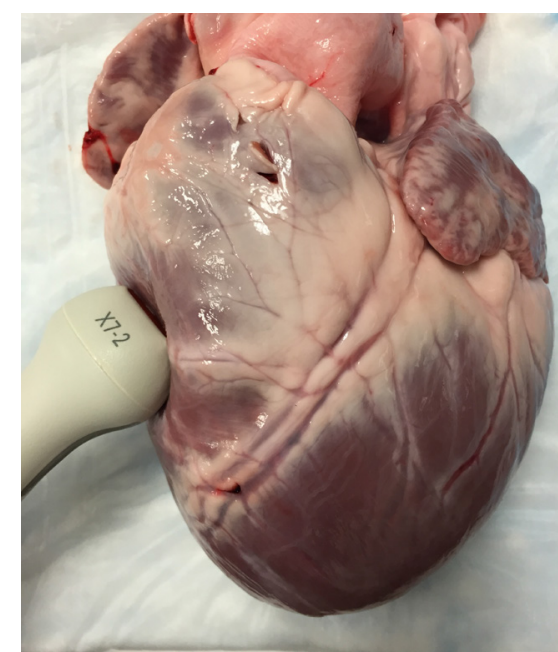

Figure 30 Epicardial left ventricle long-axis view (porcine heart).

severity, the more confident the diagnosis $(8,19)$.

TEE factors we emphasize in discussions with the surgeon when considering intervention on the TV include:

* The anatomical mechanism for the TR;

* Measuring the annular dimension in multiple views including: ME4C, TG RV inflow-outflow and the Deep TG RV inflow-outflow views;

* The presence/absence of hepatic venous systolic flow reversal;

* Tricuspid annular dimension, both absolute and normalized.

Given the dynamic nature of functional TR, and the propensity for general anesthesia to "downgrade" the degree of TR, every effort should be made to determine whether to intervene on the TV based on preoperative imaging. The operating room is not the ideal place to determine if functional TR should be repaired.

\section{Future directions and current controversies}

Despite the increased focus and interest in the TV many questions remain for both the echocardiographer and surgeon. Further studies are needed to address these controversies and determine the best practice in regards to areas of uncertainty. These include:

\section{Structure of the TV in disease}

Further study is required in delineating the size, geometry and behavior of the $\mathrm{TV}$, and determining whether the 
appearance of the TV apparatus, or the TR jet differs in particular pathological states (i.e., primary $v s$. functional). Of particular interest is the shape and behavior of the annulus, which many echocardiographers have described in differing ways. The specific nature of its complex structure and movement within the cardiac cycle remains to be conclusively determined (36).

\section{The influence and measurement of the tricuspid annulus}

Determining which echocardiographic measure is the most important in predicting progressive TR and RV dysfunction is of importance. Some hypothesize that annular dilatation, even without regurgitation may suggest an early reversible stage of RV dysfunction, whereas severe TR and RV dysfunction suggests more severe and irreversible disease.

Of particular importance is answering the question of whether annular dilatation alone is an appropriate parameter to mandate repairing the TV and if so, in which plane (anterior-posterior, septal-lateral or other) this measure should occur. There are a number of echocardiographic views in which the annulus can be measured, but which of these is the most predictive of further valve dysfunction remains uncertain.

\section{To index or not to index?}

Although the guidelines suggest annular measurements should be indexed to BSA, it is unclear whether this should be based on actual weight, ideal weight or some other parameter. It is unknown whether in patients at either extreme of BSA a number of echocardiographic parameters should be utilized in addition to clinical judgment when determining whether to operate on the TV. Or conversely, whether all patients, with an annulus greater than a certain diameter, should have it repaired.

\section{Intervention on the TV}

Surgical techniques in the management of TV disease include repair and replacement, with repair being used in the majority if patients with functional TR (37). Suture techniques for TR include surgical plication (Kay Bicuspidisation) and the DeVega suture annuloplasty (38). However, ring annuloplasty with a rigid or semi rigid partial annuloplasty band is currently regarded as the standard for surgical repair as it is associated with more effective and durable reduction in TR (39). Some authors have emphasized the importance of using an "undersized" (nominal 26 or $28 \mathrm{~mm}$ ) rigid nonplanar annuloplasty ring for optimal results (40).

The expanding availability of percutaneous techniques may also offer the potential for effective treatment of TR (41). Whether this is by control of the regurgitation alone, with a "clip" approach, with or without treatment of the dilatation (i.e., annular cinching), remains to be seen. These approaches may have a particular place in patients who have undergone previous cardiac surgery, where repeated surgery in the presence of severe TR and RV dysfunction is a high risk undertaking $(42,43)$.

Central to answering many of these controversies will be the ongoing investigation and study of the TV apparatus. Despite the growth in 3D echocardiography and other imaging modalities, a detailed understanding of the information offered by conventional echocardiographic assessment remains an essential tool for both the surgeon and echocardiographer when planning surgical intervention and assessing the success of such procedures.

The presence of these (and other) unanswered questions, the evolution in ultrasound and other imaging technologies and an increasing appreciation of the importance of TV disease indicate that significant developments in the understanding and treatment of this previously "forgotten" valve are still to come.

\section{Acknowledgements}

None.

\section{Footnote}

Conflicts of Interest: The authors have no conflicts of interest to declare.

\section{References}

1. Bruce CJ, Connolly HM. Right-sided valve disease deserves a little more respect. Circulation 2009;119:2726-34.

2. Dreyfus GD, Corbi PJ, Chan KM, et al. Secondary tricuspid regurgitation or dilatation: which should be the criteria for surgical repair? Ann Thorac Surg 2005;79:127-32.

3. Shiran A, Sagie A. Tricuspid regurgitation in mitral valve disease incidence, prognostic implications, mechanism, and management. J Am Coll Cardiol 2009;53:401-8.

4. Mahesh B, Wells F, Nashef S, et al. Role of concomitant 
tricuspid surgery in moderate functional tricuspid regurgitation in patients undergoing left heart valve surgery. Eur J Cardiothorac Surg 2013;43:2-8.

5. Hahn RT, Abraham T, Adams MS, et al. Guidelines for performing a comprehensive transesophageal echocardiographic examination: recommendations from the American Society of Echocardiography and the Society of Cardiovascular Anesthesiologists. J Am Soc Echocardiogr 2013;26:921-64.

6. Lang RM, Badano LP, Tsang W, et al. EAE/ASE recommendations for image acquisition and display using three-dimensional echocardiography. J Am Soc Echocardiogr 2012;25:3-46.

7. David TE. Functional tricuspid regurgitation: a perplexing problem. J Am Soc Echocardiogr 2009;22:904-6.

8. Lancellotti P, Moura L, Pierard LA, et al. European Association of Echocardiography recommendations for the assessment of valvular regurgitation. Part 2: mitral and tricuspid regurgitation (native valve disease). Eur J Echocardiogr 2010;11:307-32.

9. Rogers JH, Bolling SF. The tricuspid valve: current perspective and evolving management of tricuspid regurgitation. Circulation 2009;119:2718-25.

10. Ton-Nu TT, Levine RA, Handschumacher $\mathrm{MD}$, et al. Geometric determinants of functional tricuspid regurgitation: insights from 3-dimensional echocardiography. Circulation 2006;114:143-9.

11. Iskandar SB, Ann Jackson S, Fahrig S, et al. Tricuspid valve malfunction and ventricular pacemaker lead: case report and review of the literature. Echocardiography 2006;23:692-7.

12. Paranon S, Acar P. Ebstein's anomaly of the tricuspid valve: from fetus to adult: congenital heart disease. Heart 2008;94:237-43.

13. Weinberg PM. Anatomy of tricuspid atresia and its relevance to current forms of surgical therapy. Ann Thorac Surg 1980;29:306-11.

14. Nishimura RA, Otto CM, Bonow RO, et al. 2014 AHA/ ACC guideline for the management of patients with valvular heart disease: executive summary: a report of the American College of Cardiology/American Heart Association Task Force on Practice Guidelines. J Am Coll Cardiol 2014;63:2438-88.

15. Badano LP, Agricola E, Perez de Isla L, et al. Evaluation of the tricuspid valve morphology and function by transthoracic real-time three-dimensional echocardiography. Eur J Echocardiogr 2009;10:477-84.

16. Anwar AM, Geleijnse ML, Soliman OI, et al. Assessment of normal tricuspid valve anatomy in adults by real-time three-dimensional echocardiography. Int J Cardiovasc Imaging 2007;23:717-24.

17. Klein AL, Abbara S, Agler DA, et al. American Society of Echocardiography clinical recommendations for multimodality cardiovascular imaging of patients with pericardial disease: endorsed by the Society for Cardiovascular Magnetic Resonance and Society of Cardiovascular Computed Tomography. J Am Soc Echocardiogr 2013;26:965-1012 e15.

18. Kallmeyer IJ, Collard CD, Fox JA, et al. The safety of intraoperative transesophageal echocardiography: a case series of 7200 cardiac surgical patients. Anesth Analg 2001;92:1126-30.

19. Zoghbi WA, Enriquez-Sarano M, Foster E, et al. Recommendations for evaluation of the severity of native valvular regurgitation with two-dimensional and Doppler echocardiography. J Am Soc Echocardiogr 2003;16:777-802.

20. Kasper J, Bolliger D, Skarvan K, et al. Additional crosssectional transesophageal echocardiography views improve perioperative right heart assessment. Anesthesiology 2012;117:726-34.

21. Bhave NM, Ward RP. Echocardiographic assessment and clinical management of tricuspid regurgitation. Curr Cardiol Rep 2011;13:258-64.

22. Chen TE, Kwon SH, Enriquez-Sarano M, et al. Three-dimensional color Doppler echocardiographic quantification of tricuspid regurgitation orifice area: comparison with conventional two-dimensional measures. J Am Soc Echocardiogr 2013;26:1143-52.

23. Rudski LG, Lai WW, Afilalo J, et al. Guidelines for the echocardiographic assessment of the right heart in adults: a report from the American Society of Echocardiography endorsed by the European Association of Echocardiography, a registered branch of the European Society of Cardiology, and the Canadian Society of Echocardiography. J Am Soc Echocardiogr 2010;23:685713; quiz 86-8.

24. Dreyfus GD, Martin RP, Chan KMJ, et al. Functional Tricuspid Regurgitation: A Need to Revise Our Understanding. Journal of the American College of Cardiology 2015;65:2331-6.

25. Kim HK, Kim YJ, Park JS, et al. Determinants of the severity of functional tricuspid regurgitation. Am J Cardiol 2006;98:236-42.

26. Baumgartner H, Hung J, Bermejo J, et al. Echocardiographic assessment of valve stenosis: EAE/ 
ASE recommendations for clinical practice. J Am Soc

Echocardiogr 2009;22:1-23; quiz 101-2.

27. Grant $\mathrm{AD}$, Thavendiranathan P, Rodriguez LL, et al. Development of a consensus algorithm to improve interobserver agreement and accuracy in the determination of tricuspid regurgitation severity. J Am Soc Echocardiogr 2014;27:277-84.

28. Maslow AD, Schwartz C, Singh AK. Assessment of the tricuspid valve: a comparison of four transesophageal echocardiographic windows. J Cardiothorac Vasc Anesth 2004;18:719-24.

29. Mahmood F, Kim H, Chaudary B, et al. Tricuspid annular geometry: a three-dimensional transesophageal echocardiographic study. J Cardiothorac Vasc Anesth 2013;27:639-46.

30. Owais K, Taylor CE, Jiang L, et al. Tricuspid annulus: a three-dimensional deconstruction and reconstruction. Ann Thorac Surg 2014;98:1536-42.

31. Cobey FC, McInnis JA, Gelfand BJ, et al. A method for automating 3-dimensional proximal isovelocity surface area measurement. J Cardiothorac Vasc Anesth 2012;26:507-11.

32. Anwar AM, Soliman OI, Nemes A, et al. Value of assessment of tricuspid annulus: real-time threedimensional echocardiography and magnetic resonance imaging. Int J Cardiovasc Imaging 2007;23:701-5.

33. Reeves ST, Glas KE, Eltzschig H, et al. Guidelines for performing a comprehensive epicardial echocardiography examination: recommendations of the American Society of Echocardiography and the Society of Cardiovascular Anesthesiologists. Anesth Analg 2007;105:22-8.

34. Kumbharathi RB, Taneja R, Mehra R, et al. Evaluation of tricuspid and pulmonary valves using epicardial and

Cite this article as: Luxford J, Bassin L, D'Ambra M. Echocardiography of the tricuspid valve. Ann Cardiothorac Surg 2017;6(3):223-239. doi: 10.21037/acs.2017.05.15 transesophageal echocardiography--a comparative study. J Cardiothorac Vasc Anesth 2012;26:32-8.

35. Maini B. Real-time three-dimensional intracardiac echocardiography: an early single-center experience. J Invasive Cardiol 2015;27:E5-E12.

36. Knio ZO, Montealegre-Gallegos M, Yeh L, et al. Tricuspid annulus: A spatial and temporal analysis. Ann Card Anaesth 2016;19:599-605.

37. Badhwar V, Rankin JS, He M, et al. Performing Concomitant Tricuspid Valve Repair at the Time of Mitral Valve Operations Is Not Associated With Increased Operative Mortality. The Annals of Thoracic Surgery.

38. Chikwe J, Anyanwu AC. Surgical strategies for functional tricuspid regurgitation. Semin Thorac Cardiovasc Surg 2010;22:90-6.

39. Rodes-Cabau J, Taramasso M, O'Gara PT. Diagnosis and treatment of tricuspid valve disease: current and future perspectives. Lancet 2016;388:2431-42.

40. Ghoreishi M, Brown JM, Stauffer CE, et al. Undersized tricuspid annuloplasty rings optimally treat functional tricuspid regurgitation. Ann Thorac Surg 2011;92:89-95; discussion 6.

41. de Agustin JA, Viliani D, Vieira C, et al. Proximal isovelocity surface area by single-beat three-dimensional color Doppler echocardiography applied for tricuspid regurgitation quantification. J Am Soc Echocardiogr 2013;26:1063-72.

42. McCarthy PM, Bhudia SK, Rajeswaran J, et al. Tricuspid valve repair: durability and risk factors for failure. J Thorac Cardiovasc Surg 2004;127:674-85.

43. Mangoni AA, DiSalvo TG, Vlahakes GJ, et al. Outcome following isolated tricuspid valve replacement. Eur J Cardiothorac Surg 2001;19:68-73. 
Supplementary

Table S1 Echocardiographic parameters used to quantify TR severity

\begin{tabular}{|c|c|c|}
\hline Parameter & Usefulness/advantages & Limitations \\
\hline Annular diameter & Dilatation sensitive for severe TR & Dilatation seen in other conditions \\
\hline CF TR Jet & $\begin{array}{l}\text { Ease of use; evaluates the spatial orientation of TR jet; } \\
\text { good screening test for mild vs. severe TR }\end{array}$ & $\begin{array}{l}\text { Can be inaccurate for estimation of TR severity; influenced } \\
\text { by technical and hemodynamic factors; underestimates } \\
\text { eccentric jet adhering to RA wall }\end{array}$ \\
\hline PISA method & $\begin{array}{l}\text { Can be used in eccentric jets; not affected by the } \\
\text { etiology of TR or other valve leak; quantitative: } \\
\text { estimate EROA and regurgitant volume; large flow } \\
\text { convergence at } 28 \mathrm{~cm} / \mathrm{s} \text { alerts to significant TR }\end{array}$ & $\begin{array}{l}\text { PISA shape affected by: aliasing velocity, non-circular } \\
\text { orifice, systolic changes in regurgitant flow, adjacent } \\
\text { structures (flow constrainment); errors in PISA radius } \\
\text { measurement are squared; inter-observer variability; } \\
\text { validated only in a few studies }\end{array}$ \\
\hline CW TR jet profile & Simple, easily available & $\begin{array}{l}\text { Qualitative; complementary finding; complete signal difficult } \\
\text { to obtain in eccentric jet }\end{array}$ \\
\hline Hepatic vein flow & Simple; systolic flow reversal specific for severe TR & Affected by RA pressure, AF \\
\hline Peak E velocity & $\begin{array}{l}\text { Simple, easily available; usually increased in severe } \\
\text { TR }\end{array}$ & $\begin{array}{l}\text { Affected by RA pressure, AF, RV relaxation; complementary } \\
\text { finding }\end{array}$ \\
\hline RA and RV size & $\begin{array}{l}\text { Dilation sensitive for chronic severe TR; normal size } \\
\text { almost excludes severe chronic TR }\end{array}$ & $\begin{array}{l}\text { Dilatation observed in other conditions (non-specific); may } \\
\text { be normal in acute severe TR }\end{array}$ \\
\hline
\end{tabular}

Modified from (8). TR, tricuspid regurgitation; TV, tricuspid valve; EROA, effective regurgitant orifice area; PISA, proximal isovolumetric surface area; AF, atrial fibrillation. 